intraspécifiques très importantes (IANP $=30$ à 80 ) en relation avec les critères agronomiques.

\section{Composition de la carcasse et qualité de la viande chez le taurillon culard soumis à un engraissement intensif ou à une croissance faible suivie d'une croissance accélérée*. A Clinquart, JL Hornick, C Van Eenaeme, AP Mayombo, L Istasse (Service de nutrition, faculté de médecine vétérinaire, université de Liège, B43, 4000 Liège-Sart Tilman, Belgique)}

L'influence d'une réduction de la vitesse de croissance avant engraissement a été déterminée sur la composition de la carcasse et la qualité de la viande de taureaux Blanc-Bleu-Belge culards. Dix taureaux témoins ( $T$ ) ont été engraissés durant $252 \mathrm{j}$ à raison de $1,3 \mathrm{~kg} / \mathrm{j}$ et abattus à 630 $\mathrm{kg}$. Dix taureaux $(\mathrm{CF})$ ont réalisé $0,6 \mathrm{~kg} / \mathrm{j}$ durant $115 \mathrm{j}$, puis $1,6 \mathrm{~kg} / \mathrm{j}$ durant $174 \mathrm{j}$; ils ont été abattus à $632 \mathrm{~kg}$. Les animaux ont été abattus lorsque la moyenne des gains journaliers, calculée après 4 pesées consécutives à 1 sem d'intervalle descendait en-dessous de $1 \mathrm{~kg} / \mathrm{j}$. Les poids de carcasses ont été de 409 et $401 \mathrm{~kg}$ dans les groupes $\mathrm{T}$ et $\mathrm{CF}$ respectivement. $\mathrm{Le} \mathrm{pH}$ et la température ont été mesurés 1,2 et $4 \mathrm{~h}$ post mortem. Le segment tricostal 7-8-9 a été prélevé $48 \mathrm{~h}$ post mortem afin de déterminer la composition de la carcasse et les paramètres de qualité du Longissimus Thoracis. La couleur de la viande a été déterminée par le système CIE $L^{*} a^{*} b^{*}, L^{*}$ exprimant la luminosité, $a^{\star}$ et $b^{\star}$ la teinte. La tendreté a été estimée par la force maximale de cisaillement. Une légère augmentation de la proportion de tissu adipeux a été observée dans le groupe CF $(13,4 \pm 1,4$ vs $12,4 \pm 1,4 \% ; P<0,1)$ au détriment de la proportion de muscle $(73,1 \pm$ 2,0 vs $74,7 \pm 1,4 \%$ ). L'évolution du $\mathrm{pH}$ et de la température post mortem a été similaire dans les 2 groupes. Le $\mathrm{pH}$ final était respectivement de $5,48 \pm 0,19$ vs $5,55 \pm 0,11$ dans les groupes CF et $T$. Aucune différence significative n'a été trouvée dans la qualité de la viande. Certaines tendances ont néanmoins été observées pour la couleur, la capacité de rétention d'eau et la tendreté. La viande était plus foncée et plus rouge dans le groupe CF $(41,2 \pm 2,7$ vs $42,5 \pm 2,4 \%$ pour $L^{*} ; 16,9 \pm 1,1$ vs $16,2 \pm 2,4$ pour $\left.a^{*}\right)$. La perte de jus mesurée durant une période de $7 \mathrm{j}$ a été de $3,5 \pm 0,8$ vs $4,3 \pm 1,0 \%$ dans les groupes
$\mathrm{CF}$ et $\mathrm{T}$. Les forces de cisaillement ont été plus faibles dans le groupe CF $(33,6 \pm 8,0$ vs $40,3 \pm$ $12,6 \mathrm{~N})$. Une seule tendance défavorable a été plus faible dans le groupe CF pour les pertes lors d'une cuisson à $75^{\circ} \mathrm{C}$ durant $50 \min (22,1 \pm 2,6 \%$ vs $21,0 \pm 4,8 \%$ ). En conclusion, un allongement de la durée de croissance et une diminution du gain quotidien moyen total dans le groupe CF ont légèrement augmenté la proportion de graisse dans la carcasse, mais n'ont pas altéré la qualité de la viande.

*Recherches subventionnées par I'IRSIA, rue de Crayer, 6, B-1050 Bruxelles, Belgique.

\section{Extensification de la production de viande bovine en zone herbagère nor- mande. D Dozias ${ }^{1}$, D Micol ${ }^{2}$, JR Peccatte ${ }^{1}$ ( INRA, domaine SEA du Pin au Haras, 61310 Exmes; 2 INRA-Theix, 63122 Saint- Genès-Champanelle, France)}

Dans les conditions actuelles de l'agriculture, les élevages laitiers des zones herbagères de l'Ouest dégagent des moyens de production, notamment des surfaces en herbe. Une action de recherche, conduite au domaine SEA du Pin au Haras (61), envisage la valorisation de ces surfaces par l'intermédiaire d'une production complémentaire de viande bovine à partir de bœufs conduits essentiellement avec de l'herbe du jeune âge à l'abattage.

Deux voies sont explorées par une étude globale des systèmes fourrager et d'élevage : i) l'exploitation intensive des prairies (système 1) en recherchant la production maximale de viande par hectare d'herbe (bovins de 2 ans), associée à un niveau important d'intrants (fertilisation azotée, coûts des récoltes, aliments complémentaires) ; ii) l'utilisation plus libérale des mêmes surfaces (système E) conduisant à une extensification de la gestion des ressources et à une diminution des intrants (bovins de 33 mois, récoltes en foin, pâturage simplifié, fertilisation azotée quasi nulle).

Trois séries annuelles d'animaux normands ( $n=144$ ) ont été étudiées au cours de 5 années de suivi. Les carcasses atteignent un poids de $363 \pm 17 \mathrm{~kg}$ à 24,5 mois dans le système I et $402 \pm 25 \mathrm{~kg}$ à 32,5 mois dans le système $E$ sans que cet écart se traduise par des différences notables de composition corporelle et de caractéristiques des muscles. 